\title{
Effect of sodium sulfadimethylpyrimidine on multiple forms of cytochrome P450 in chicken
}

\author{
S. S. Adav', P. A. Padmawar², S. P. Govindwar ${ }^{3}$
}

${ }^{1}$ Department of Agricultural Chemistry, National Taiwan

University, Taipei, Taiwan 10617, ROC; ${ }^{2}$ Department of Chemistry, University of Massachusetts Lowell, Lowell, MA, USA;

${ }^{3}$ Department of Biochemistry, Shivaji University, Kolhapur, India

Received: 17.7.2004

Revised: 23.11.2004

Accepted: 4.12.2004

Correspondence to:

S. P. Govindwar

E-mail:

spgovindwar@rediffmail.com; spg_biochem@unishivaji.ac.in

\begin{abstract}
Objective: To study the effect of sodium sulfadimethylpyrimidine (SDMP) on different forms of CYP 450 enzymes induced by phenobarbital (CYP 2B1, $2 B 2$ and 3A), isoniazid (CYP 2E1), benzo(a)pyrene (CYP 1A1), clotrimazole (CYP 3A), and clofibrate (CYP 4A).

Materials and Methods: Chickens (Hubbard, male) weighing 250-300 g were divided into 17 groups of six each. Five experimental sets were prepared containing three subgroups each to test five different inducers. Microsomes were isolated by calcium precipitation. The levels of electron transport components, CYP 450, cytochrome b5, and cytochrome creductase were determined using extinction coefficients. Activities of drug-metabolizing enzymes were assayed.

Results: All inducers (phenobarbital, isoniazid, benzo(a)pyrene, clotrimazole, and clofibrate) showed significant induction of mixed function oxidase in chicken. The SDMP treatment of inducer-pretreated chicken caused a significant decrease in electron transport components and activities of drug-metabolizing enzymes when compared with treatment of inducer alone. Phenobarbital, isoniazid, and benzo(a)pyrene treatments of SDMP-pretreated chicken showed no significant change in induction pattern, however, significant alterations were observed in the induction pattern of clotrimazole and clofibrate.

Conclusion: Our studies suggest that CYP 2B1, 2B2, 3A; CYP 2E1; CYP 1A1; CYP 3A and CYP 4A are susceptible species of CYP 450 to SDMP and its metabolites. The SDMP also affected in the induction pattern of some of the inducers with respect to CYP 450 isoforms.
\end{abstract}

KEY WORDS: Aniline hydroxylase; aminopyrine N-demethylase; CYP 450; mixed function oxidase; sodium; sulfadimethylpyrimidine.

\section{Introduction}

Microsomal cytochrome P450 (CYP 450) consists of a multigene family that plays an important role in the metabolism of a wide variety of endogenous compounds and xenobiotics including drugs, carcinogens, toxic chemicals, steroids, and fatty acids. ${ }^{[1]}$ These liver microsomal CYP 450 isozymes exhibit broad, often overlapping specificities towards their substrates and are differentially regulated by several factors including sex, age, and administration of drugs. ${ }^{[2]-[6]}$ Depending on the isozymes and substrate, different forms of CYP 450 may catalyze the metabolism of a particular substrate at comparable rates but biotransform other substrates at significantly different rates. Rat CYP 450 b and rabbit LM2 catalyze the $N$-demethylation of benzphetamine 100 times faster than other enzymes. ${ }^{[5]}$ Rat liver CYP 450 a, 450 b, and
450 c preferentially hydrolyze testosterone at the $7 \alpha, 17 \alpha$, and $6 \beta$ positions, respectively. The available evidence indicates that the presence of different CYP 450 isozymes in tissues from various sources is primarily responsible for the observed differences in metabolism. The difference in substrate specificity, regiospecificity, and stereospecificity of various CYP 450 isozymes plays an important role in regulation pathways of a given drug. Thus, the relative proportions of various forms of CYP 450 in a given tissue or individual is an important factor in determining the cytotoxic and carcinogenic action of many toxic compounds. Sodium sulfadimethylpyrimidine (SDMP) is routinely used for acute coccidiosis, coryza, cholera, pullorum, and antipestifer diseases in chicken. ${ }^{[7]}$ The SDMP influences mixed function oxidase in chicken and it depends on the dose, duration of SDMP treatment and strain of chicken. ${ }^{[8]}$ The SDMP 
(300 mg/kg, body weight) treatment to chicken decreases the levels of electron transport components and drug-metabolizing enzymes. In vitro addition of SDMP shows the inhibitory action on CYP 450. ${ }^{[8]}$ However, the effect of SDMP on various forms of CYP 450 is still unknown, hence the present work was undertaken to study the effect of SDMP on different forms of CYP 450 enzymes.

\section{Materials and methods}

\section{Animals}

One day old healthy male chickens $(n=102$, BW $=250$ $300 \mathrm{~g}$ ) of Hubbard strain were obtained from Nicholas Breeders Pvt. Ltd., Tamgaon, Kolhapur. The chickens were caged in to wire bottom cages and grown to 3 weeks with poultry feed (chick mesh) and tap water ad libitum. Chickens were divided into 17 subgroups containing six birds each.

\section{Chemicals}

Nicotinamide adenine dinucleotide phosphate (reduced form, NADPH), nicotinamide adenine dinucleotide phosphate (NADP), cytochrome c, glucose 6-phosphate, glucose 6phosphate dehydrogenase, aminopyrine, aniline hydrochloride, $N$-2-Hydroxymethyl Piperazine- $N$-2 Ethane Sulphonic acid (HEPES), benzo(a)pyrene, clotrimazole, clofibrate, and isoniazid were obtained from Sigma Chemicals Co. (St. Louis, MO, USA). Phenobarbital was obtained from John Baker (Colorado Springs, CO). The SDMP(100\% purity) was obtained from Cyanamid India Ltd., (Valsad, India). Standard kits of alanine transferase, aspartate transferase, sucrose, phenol, trichloroacetic acid, sodium chloride, potassium chloride, calcium chloride, and other chemicals of analytical grade were obtained from Qualigens Chemical (Bombay, India).

\section{Treatment of animals}

The treatment details are provided in the tables. Chickens were injected (i.p.) daily between 7.30 and 8.30 a.m. Group 1 was injected with $0.9 \%$ saline and served as control. Group 2 was treated with standard dose of SDMP (300 mg/kg, i.p., single dose). Five experimental sets were prepared containing three subgroups each to test five different inducers and SDMP effects on pre and post-treated inducers group. All animals were killed $24 \mathrm{~h}$ after the last treatment.

\section{Preparation of microsomes}

The chickens used in this study were decapitated and their livers were removed rapidly, weighed, and perfused with icecold $1.15 \% \mathrm{KCl}$ solution containing $0.05 \mathrm{mM}$ EDTA. Each of the perfused liver was homogenized with two volumes of icecold $0.25 \mathrm{M}$ sucrose in Potter Elvehjem homogenizer. The homogenate was centrifuged at $10,000 \mathrm{X} \mathrm{g}$ for $30 \mathrm{~min}$ in a refrigerated centrifuge (Remi C-24). Microsomes from the supernatant fraction were isolated by the procedure of Cinti et al. ${ }^{[9]}$ The microsomal pellet was washed once with $1.15 \%$ KCl solution containing $0.05 \mathrm{mM}$ EDTA, resuspended in phosphate buffer ( $\mathrm{pH}$ 7.4) and the suspension was used for microsomal enzyme assays. Microsomal protein was estimated by the Biuret method, ${ }^{[10]}$ using bovine serum albumin as the standard.

\section{Microsomal enzyme assays}

The levels of microsomal electron transport components, CYP 450, and cytochrome b5 were determined using a Shimadzu-UV-Visible recording spectrophotometer (UV-160A) by the procedure of Omura and Sato. ${ }^{[1]}$ The NADPH-cytochrome c-reductase was determined by the method of Masters et al. ${ }^{[12]}$ Aminopyrine $N$-demethylase activity was assayed according to the procedure of Schenkman et al. ${ }^{[13]}$ Formaldehyde liberated during $N$-demethylation was estimated by the procedure of Nash. ${ }^{[14]}$ Aniline hydroxylase assay was performed using the procedure of Govindwar and Dalvi. ${ }^{[15]}$

Statistical analysis

Data analysis was performed using the one-way analysis of variance (ANOVA) and Tukey-Kramer multiple comparison tests and unpaired $t$ test. $P<0.05$ were considered significant. Values for all parameters were compared with the control (untreated) group. Further, groups of pre and post-treatment of SDMP were compared with the group of respective inducer treatment.

\section{Results}

Administration of SDMP showed significant decrease in electron transport components and drug-metabolizing enzymes as observed earlier. ${ }^{[9]}$ Phenobarbital, isoniazid, benzo(a)pyrene treatments showed significant induction of microsomal protein, however, treatment with clotrimazole and clofibrate did not show any significant change. Significant increases were observed in electron transport components and drugmetabolizing enzymes due to phenobarbital, isoniazid, benzo(a)pyrene, clotrimazole, and clofibrate, except cytochrome c-reductase in benzo(a)pyrene treatment (Tables 1 and 2).

The SDPM treatment of phenobarbital, isoniazid, clotrimazole or clofibrate pretreated chicken did not change the level of microsomal proteins except in benzo(a)pyrene, when compared with respective control. However, significant decrease in CYP 450, cytochrome b5, cytochrome c-reductase, aminopyrine $\mathrm{N}$-demethylase, and aniline hydroxylase was observed when compared with the respective control groups (Tables 1 and 2).

Inducer (phenobarbital, benzo(a)pyrene or clotrimazole) treatment of SDMP pretreated chicken showed no significant change in electron transport components and drugmetabolizing enzymes when compared with respective control (inducer treatment alone), except, SDMP treatment prior to clotrimazole resulted in a significant increase in the activities of cytochrome c-reductase and aniline hydroxylase, when compared to clotrimazole alone. Isoniazid treatment of SDMP pretreated chicken caused significant decrease in microsomal protein, CYP 450, cytochrome b5, and aminopyrine $N$ demethylase when compared to isoniazid treatment alone. Clofibrate treatment after SDMP pretreatment also resulted in noticeable increase in cytochrome b5, CYP 450, and aniline hydroxylase when compared with control. A significant decrease in cytochrome b5, CYP 450, and cytochrome creductase was observed due to clofibrate treatment of SDMP pretreated chicken when compared with clofibrate treatment alone (Tables 1 and 2).

A significant inhibition (of 21-61\% and 13-27\%) of aminopyrine $N$-demethylase, and aniline hydroxylase activities was observed due to in vitro addition of SDMP (4.2 mM) in SDMP, phenobarbital, benzo(a)pyrene, isoniazid, clotrimazole, and clofibrate-treated chicken liver microsomes (data not shown). 


\section{Table 1}

Alteration in liver microsomal protein and electron transport components due to SDMP (300 mg/kg, i.p., for 1 day), phenobarbital (80 mg/kg, i.p., for 3 days), isoniazid (100 mg/kg, i.p., for 3 days), benzo(a)pyrene (20 mg/kg, i.p., for 2 days), clotrimazole (100 mg/kg, i.p., for 3 days), and clofibrate $(100 \mathrm{mg} / \mathrm{kg}$, i.p., for 3 days) treatment in Hubbard (male) chicken

\begin{tabular}{|c|c|c|c|c|}
\hline Group & Microsomal protein ${ }^{\dagger}$ & Cytochrome b5 & Cytochrome $\mathrm{P}^{450^{\ddagger}}$ & Cytochrome c-reductase ${ }^{\mathcal{S}}$ \\
\hline SDMP & $15.10 \pm 0.43$ & $0.15 \pm 0.01^{* * *}$ & $0.19 \pm 0.01^{* *}$ & $42.00 \pm 0.50^{* * *}$ \\
\hline Phenobarbital & $24.00 \pm 1.20^{* * *}$ & $0.37 \pm 0.01^{* * *}$ & $0.52 \pm 0.01^{\star * *}$ & $70.72 \pm 0.80^{* * *}$ \\
\hline Phenobarbital + SDMP & $26.00 \pm 0.14^{* * *}$ & $0.31 \pm 0.01^{* * *, \$ \$}$ & $0.40 \pm 0.01^{* * *, \$ \$ \$}$ & $68.00 \pm 0.72^{* * *}$ \\
\hline SDMP + Phenobarbital & $22.00 \pm 0.33^{* * *}$ & $0.35 \pm 0.01^{* * *}$ & $0.56 \pm 0.01^{* * *}$ & $75.91 \pm 0.88^{* * *, \$}$ \\
\hline Isoniazid & $25.30 \pm 0.79^{* * *}$ & $0.52 \pm 0.01^{* * *}$ & $0.56 \pm 0.02^{* * *}$ & $94.00 \pm 1.29^{* * *}$ \\
\hline Isoniazid + SDMP & $23.30 \pm 0.64^{\star \star \star}$ & $0.28 \pm 0.01^{* *, \$ \$ \$}$ & $0.30 \pm 0.01^{\$ \$ \$}$ & $59.50 \pm 1.28^{\$ \$ \$}$ \\
\hline SDMP + Isoniazid & $19.00 \pm 0.33^{*, \$ \$ \$}$ & $0.40 \pm 0.02^{* * * \$ \$ \$}$ & $0.50 \pm 0.01^{* * \star \$}$ & $91.80 \pm 1.14^{* * *}$ \\
\hline \multicolumn{5}{|c|}{ SDMP effect on benzo(a)pyrene pre and post-treated groups } \\
\hline Benzo(a)pyrene & $22.20 \pm 0.20^{\star * *}$ & $0.28 \pm 0.01^{* *}$ & $0.49 \pm 0.01^{* * *}$ & $61.87 \pm 0.58$ \\
\hline Benzo(a)pyrene + SDMP & $29.00 \pm 0.30^{* * *, \$ \$ \$}$ & $0.18 \pm 0.01^{\$ \$ \$}$ & $0.40 \pm 0.01^{* * *, \$ \$ \$}$ & $50.62 \pm 0.40 * * * \$ \$ \$$ \\
\hline Clotrimazole + SDMP & $17.00 \pm 0.70$ & $0.25 \pm 0.01^{\$ \$ \$}$ & $0.30 \pm 0.01 \$ \$$ & $53.38 \pm 1.28^{* * \star, \$ \$ \$}$ \\
\hline SDMP + Clotrimazole & $12.50 \pm 0.33^{*}$ & $0.34 \pm 0.01^{* * *}$ & $0.48 \pm 0.01^{* * *}$ & $97.38 \pm 1.14^{* * *, \$ \$ \$}$ \\
\hline \multicolumn{5}{|c|}{ SDMP effect on clofibrate pre and post-treated groups } \\
\hline Clofibrate & $16.00 \pm 0.05$ & $0.38 \pm 0.01^{* * *}$ & $0.50 \pm 0.01^{* * *}$ & $80.62 \pm 1.14^{\star * *}$ \\
\hline Clofibrate + SDMP & $13.50 \pm 0.48$ & $0.26 \pm 0.01^{\$ \$ \$}$ & $0.28 \pm 0.01^{\$ \$ \$}$ & $58.00 \pm 0.77^{\$ \$ \$}$ \\
\hline SDMP + Clofibrate & $16.50 \pm 0.40$ & $0.28 \pm 0.01^{* *, \$ \$ \$}$ & $0.32 \pm 0.01 * \$ \$ \$$ & $60.00 \pm 0.48^{\$ \$ \$}$ \\
\hline \multirow[t]{2}{*}{ One-way ANOVA } & 97.101 & 85.816 & 115.38 & 309.5 \\
\hline & $<0.0001$ & $<0.0001$ & $<0.0001$ & $<0.0001$ \\
\hline
\end{tabular}

Values represent mean \pm SEM. $n=6$ in each group. $\mathrm{df}=86,12 .{ }^{*} P<0.05,{ }^{* \star} P<0.01,{ }^{* \star *} P<0.001$ Vs control and $\$ P<0.05, \$ \$ P<0.01,{ }^{\$ \$} P<0.001 \mathrm{Vs}$ respective inducer group (Tukey-Kramer multiple comparisons test). ${ }^{\top} \mathrm{mg}$ protein $/ \mathrm{g}$ liver. ${ }^{\ddagger} \mathrm{nmol} / \mathrm{mg}$ microsomal protein. ${ }^{\circ} \mathrm{nmol}$ of cytochrome $\mathrm{c}$ reduced/min/mg $\mathrm{microsomal} \mathrm{protein}$.

\section{Discussion}

The SDMP is used as a marker of $N$-acetylation in man for genetic determination of the $\mathrm{N}$-acetyl transferase activity. ${ }^{[16]-}$ ${ }^{\text {[18] }}$ Other than acetylation, these drugs can be metabolized by glucuronidation, sulfation, and oxidation to hydroxylamine, which depends on species and physiological conditions. ${ }^{[19],[20]}$ Our earlier investigation showed biphasic response of SDMP, inhibition of catalytic and spectral activity of CYP 450 due to addition of SDMP in vitro. ${ }^{[8]}$ No alteration in phenobarbital sleeping time in SDMP-treated rats and differential induction pattern in different strains of chicken ${ }^{[8]}$ encouraged us to study the effect of SDMP on various CYP 450 isoforms. The main objective was to study the effect on phenobarbital induced CYP 2B1, 2B2, 3A; isoniazid induced CYP 2E1; benzo(a)pyrene induced CYP 1A1; Clotrimazole induced CYP 3A and clofibrate induced CYP 4A microsomal CYP 450.

The protein levels and other parameters of mixed function oxidase system were increased following phenobarbital treatment. The CYP $2 \mathrm{~B} 3$ is not induced by phenobarbital, whereas CYP 450 2B1, 2B2 are phenobarbital inducible. Indeed, it seems the phenobarbital induction is not limited to CYP 450, in so far as several other enzymes that contribute to foreign compound metabolism including aldehyde dehydrogenase, epoxide hydroxylase, NADPH-cytochrome P450 reductase, UDP-glucuronosyl transferase and several glutathione s-transferase are inducible by Phenobarbital. ${ }^{[21 \mid-[23]}$ The effect of phenobarbital, unlike those 3-methylcholanthrene, include proliferation of smooth endoplasmic reticulum, stimulation of liver weight gain, liver tumor promotion, and general stabilization of liver microsomal protein and are thus pleiotropic. ${ }^{[24]}$

No alteration in the levels of CYP 450 and drug-metabolizing enzymes due to pretreatment of SDMP indicates any significant effect of SDMP or it's metabolites on the induction mechanism of phenobarbital. Hence there is no alteration in phenobarbital sleeping time in chicken. Isoniazid was found to be a strong inducer of mixed function oxidase system.

Benzo(a)pyrene treatment of chickens showed a significant increase in microsomal proteins, electron transport components and drug-metabolizing enzymes which is usually observed in rats. ${ }^{\mid 25]}$ The SDMP treatment of benzo(a)pyrene pretreated chickens showed significant decrease in the electron transport components and drug-metabolizing enzymes which indicates susceptibility of CYP 1A1 to SDMP. Benzo(a)pyrene treatment of SDMP pretreated chicken showed no change in CYP 450, cytochrome c-reductase, and the activity of aniline hydroxylase when compared with benzo(a)pyrene treatment alone. However, a significant decrease in aminopyrine $N$ - 
Table 2

Alteration in chicken liver microsomal drug-metabolizing enzymes due to SDMP (300 $\mathrm{mg} / \mathrm{kg}$, i.p., for 1 day), phenobarbital ( $80 \mathrm{mg} / \mathrm{kg}$, i.p., for 3 days), isoniazid ( $100 \mathrm{mg} / \mathrm{kg}$, i.p., for 3 days), benzo(a)pyrene (20 mg/kg, i.p., for 2 days), clotrimazole (100 mg/ kg, i.p., for 3 days), and clofibrate (100 $\mathrm{mg} / \mathrm{kg}$, i.p., for 3 days) treatment in hubbard (male) chicken

\begin{tabular}{|c|c|c|}
\hline Group & $\begin{array}{c}\text { Aminopyrine } \\
\text { N-demethylase } t\end{array}$ & $\begin{array}{c}\text { Aniline } \\
\text { hydroxylase }^{\ddagger}\end{array}$ \\
\hline Control & $3.95 \pm 0.05$ & $1.29 \pm 0.04$ \\
\hline SDMP & $2.90 \pm 0.05^{\star * *}$ & $1.06 \pm 0.02$ \\
\hline \multicolumn{3}{|c|}{ SDMP effect on phenobarbital pre and post-treated groups } \\
\hline Phenobarbital & $6.31 \pm 0.07^{\star * *}$ & $1.56 \pm 0.04$ * \\
\hline Phenobarbital + SDMP & $2.80 \pm 0.10^{* * *, \$ \$ \$}$ & $1.180 \pm 0.08^{\$ \$ \$}$ \\
\hline SDMP + Phenobarbital & $5.94 \pm 0.45^{* * *}$ & $1.54 \pm 0.02$ \\
\hline \multicolumn{3}{|c|}{ SDMP effect on isoniazid pre and post-treated groups } \\
\hline Isoniazid & $5.62 \pm 0.20^{* * *}$ & $2.12 \pm 0.02^{\star \star *}$ \\
\hline Isoniazid + SDMP & $3.95 \pm 0.05^{\$ \$ \$}$ & $1.49 \pm 0.04^{\$ \$}$ \\
\hline SDMP + Isoniazid & $4.80 \pm 0.09^{*, \$}$ & $2.59 \pm 0.02^{* * *, \$ \$ \$}$ \\
\hline \multicolumn{3}{|c|}{ SDMP effect on benzo(a)pyrine pre and post-treated groups } \\
\hline Benzo(a)pyrine & $4.96 \pm 0.15^{\star \star}$ & $1.76 \pm 0.02^{* * *}$ \\
\hline Benzo(a)pyrine + SDMP & $2.80 \pm 0.15^{\star \star \star \star \$ \$ \$}$ & $1.10 \pm 0.04^{\$ \$ \$}$ \\
\hline SDMP + Benzo(a)pyrine & $4.09 \pm 0.13^{\$}$ & $1.85 \pm 0.08^{* \star *}$ \\
\hline \multicolumn{3}{|c|}{ SDMP effect on clotrimazole pre and post-treated groups } \\
\hline Clotrimazole & $4.90 \pm 0.09^{\star *}$ & $1.73 \pm 0.04^{\star * *}$ \\
\hline Clotrimazole + SDMP & $3.95 \pm 0.04^{\$ \$}$ & $1.28 \pm 0.07^{\$ \$ \$}$ \\
\hline SDMP + Clotrimazole & $4.65 \pm 0.11$ & $2.00 \pm 0.08^{* * * \$}$ \\
\hline \multicolumn{3}{|c|}{ SDMP effect on clofibrate pre and post-treated groups } \\
\hline Clofibrate & $4.90 \pm 0.08^{* *}$ & $2.44 \pm 0.04^{* * *}$ \\
\hline Clofibrate + SDMP & $3.95 \pm 0.10^{\$ \$}$ & $2.00 \pm 0.02^{* * *, \$ \$ \$}$ \\
\hline SDMP + Clofibrate & $4.40 \pm 0.10$ & $2.22 \pm 0.08^{\star \star \star}$ \\
\hline \multirow[t]{2}{*}{ One-way ANOVA } & 46.649 & 86.541 \\
\hline & $<0.0001$ & $<0.0001$ \\
\hline
\end{tabular}

Values represent mean \pm SEM. $n=6$ in each group. $\mathrm{df}=86,12 .{ }^{*} P<0.05 .{ }^{*} P$ $<0.01,{ }^{* * *} P<0.001$ vs control and $\$ P<0.05, \$ \$ P<0.01,{ }^{\$} \$ \$<0.001$ vs respective inducer group (Tuckey-Kramer multiple comparisons test). ${ }^{\dagger} \mathrm{nmol}$ formaldehyde liberated/min per mg microsomal protein. ${ }^{\ddagger} \mathrm{nmol} p$-aminophenol formed/min per mg microsomal protein.

demethylase may be because of partial destruction of CYP 450 due to residual SDMP or it's metabolites.

Clotrimazole is a specific inducer of CYP 3A activity. ${ }^{[2]}$ Posttreatment of SDMP to clotrimazole pretreated chicken showed a significant decrease in electron transport components and drug-metabolizing enzymes. This indicates that CYP $3 \mathrm{~A}$ is sensitive to SDMP and it's metabolites. As can be seen from the prior treatment of SDMP to clotriamazole-treated chicken, further increase in the activities of cytochrome c-reductase, and aniline hydroxylase indicates the enhancement of inductive mechanism.

A unique induction pattern from clofibrate treatment has been observed previously. ${ }^{[27]}$ To observe the effect of SDMP or it's metabolites on CYP 4A activity, the chicken were injected with a well-known inducer clofibrate. Clofibrate treatment to male chicken showed significant increase in the electron transport components and drug-metabolizing enzymes. Clofibrate treatment prior to SDMP treatment resulted in significant de- crease in the levels of electron transport components and drugmetabolizing enzymes compared with clofibrate treatment alone. This indicates the destruction of CYP 4A is due to SDMP or it is metabolites. It is surprising to note significant low levels of electron transport components with insignificant changes in the activities of drug-metabolizing enzymes due to posttreatment of clofibrate to SDMP-treated chicken when compared with the clofibrate treatment alone. It clearly indicates that the alteration in the inductive mechanism of clofibrate.

In vitro addition of SDMP (4.2 mM) in microsomal incubates obtained from various inducer treatments showed different percent inhibition in aminopyrine $N$-demethylase, and aniline hydroxylase activity. It is also reported that of aminopyrine $N$-demethylase is inhibited more than aniline hydroxylase activity, which indicate that the inhibition is depending on the composition of isozymes of CYP 450. With consideration of overlapping substrate specificity and multiplicity of CYP 450, it can be concluded that CYP 2E1 might be a resistant species. The CYP 2B1, CYP 2B2, 3A, and CYP $1 \mathrm{~A} 1$ forms were found to be more susceptible.

In conclusion, this investigation indicates that all inducers viz., phenobarbital, isoniazid, benzo(a)pyrene, clotrimazole, and clofibrate of mixed function oxidase in rodents also show induction in avian system. Significant decrease in electron transport components and levels of drug-metabolizing enzymes due to SDMP post-treatment indicates the susceptibility of CYP 2B1, CYP 2B2, 3A; CYP 2E1; CYP 1A1; CYP 3A and CYP 4A to SDMP or it's metabolites. The SDMP does not have significant effect on induction pattern of phenobarbital, isoniazid, and benzo(a)pyrene. However, it certainly modulates the induction pattern with respect to isoforms of CYP 450 in case of clotrimazole, and clofibrate.

\section{References}

1. Omura T, Sato R, Cooper DY, Rosenthal O, Estabrook RW. Function of cytochrome P450 of microsomes. Fed Proc 1965;24:1181-9.

2. Thomas PE, Lu AY, Ryan D, West SB, Kawalek J, Levin W. Multiple forms of rat liver cytochrome P450. Immunochemicals evidence with antibody against cytochrome P488. J Biol chem 1976;251:1385-91.

3. Guengerich FP. Separation and purification of multiple forms of microsomal cytochrome P450. Activities of different forms of cytochrome P450 towards several compounds of environmental interest. J Biol Chem 1977;252:3970-9

4. Guengerich FP. Separation and purification of multiple forms of microsomal cytochrome P-450. Partial characterization of three apparently homogeneous cytochromes P-450 prepared from livers of phenobarbital- and 3methylcholanthrene-treated rats. J Biol Chem 1978;253:7931-9.

5. Ryan DE, Thomas PE, Korzeniowski D, Levin W. Separation and characterization of highly purified forms of liver microsomal cytochrome P450 from rats treated with polychlorinated biphenyls, Phenobarbital and 3methylcholanthrene J Biol Chem 1979;254:1365-74.

6. Guengerich FP. Isolation and purification of cytochrome P450 and existence of multiple forms. Pharmacol Ther 1979;6:99-121.

7. Daykin PW. The sulphonamides. In: Veterinary applied pharmacology and therapeutics, belfast: Northern Ireland: University Press; 1960.

8. Govindwar SP, Adav SS. Effect of sodium sulfadimethylpyrimidine on mixed function oxidase in chicken. Indian J Pharmacol 2003;35:92-8.

9. Cinti DL, Moldeus P, Schenkman JB. Kinetic parameters of drug-metabolizing enzymes in $\mathrm{Ca}^{2+}$-sedimented microsomes from rat liver. Biochem Pharmacol 1972;21:3249-56.

10. Gornall AG, Bardawill CJ, David MM. Determination of serum proteins by means of the biuret reaction. J Biol Chem 1949;177:751-66.

11. Omura T, Sato R. The carbon monoxide-binding pigment of liver microsomes. 
I. Evidence for its hemoprotein nature. J Biol Chem 1964;239:2370-8.

12. Masters BSS, William $\mathrm{CH}, \mathrm{Kamin} \mathrm{H}$. The preparation and properties of microsomal TPNH cytochrome c-reductase from pig liver. In: Estabrook RW, Pullman ME, editors. Method in Enzymology. New York: Academic Press; 1967.

13. Schenkman JB, Remmer H, Estabrook RW. Spectral studies of drug interation with hepatic microsomal cytochrome. Mol Pharmacol 1967;3:113-23.

14. Nash T. The colorimetric estimation of formaldehyde by means of the Hantzsch reaction. Biochem J 1953:55:416-21.

15. Govindwar SP, Dalvi RR. Age-dependent toxicity of acorn extract in young and old male rats. Vet Hum Toxicol 1990;32:23-6.

16. Peters JH, Gordon GR, Karat AB. Polymorphic acetylation of the antibacterials, sulfamethazine and dapsone, in South Indian subjects. Am J Trop Med Hyg 1975;24:641-8.

17. Evans DA. An improved and simplified method of detecting the acetylator phenotype. J Med Genet 1969;6:405-7.

18. White TA, Evans DA. The actylation of sulfamethazine and sulfamethoxypyridazine by human subjects. Clin Pharmacol Ther 1968;9:80-8.

19. Nouws JFM, Vree TB, Breukink HJ, Van Miert ASJPAM, Grondel J. Pharmacokinetics, hydroxylation and acetylation of sulfamethazine in mammals, birds, fish, reptiles and mollusks. In: Van Niert ASJPAM, Bogaert MG, Debackere, editors. Lancaster: MTP Press Ltd; 1986.

20. Vree TB, Hekster YA, Nouws JFM, Baakman M. Pharmacokinetics, metabolism, and renal excretion of sulfadimidine and its N4-acetyl and hydroxy metabolites in humans. Ther Drug Monit 1986;8:434-9.

21. Dunn TJ, Koleske AJ, Lindahl R, Pilot HC. Phenobarbital inducible aldehyde dehydrogenase in the rat. CDNA sequence and regulation of the mRNA by phenobarbital in responsive rats. J Biol Chem 1989;264:13057-65.

22. Pickett CB, Jeter RL, Marin J, Lu AY. Electroimmunochemical quantitation of cytochrome P450, cytochrome P488, and epoxide hydrolase in rat liver microsomes. J Biol Chem 1981;256:8815-20.

23. Kuriyama $Y$, Omura T, Siekevitz P, Palade GE. Effects of phenobarbital on the synthesis and degradation of the protein components of rat liver microsomal membranes. J Biol Chem 1969;244:2017-26.

24. Miller JA. Carcinogenesis by chemicals: an overview G.H.A. Clowes memorial lecture. Cancer Res 1970;30:559-76

25. Kodam KM, Adav SS, Govindwar SP. Effect of sulfamethazine on phenobarbital and benzo(a)pyrene induced hepatic microsomal mixed function oxidase system in rats. Toxicol Lett 1996;87:25-30.

26. Kobayashi $Y$, Matsuura Y, Kotani E, Fukuda T, Aoyagi T, Tobinaga S, et al. Structural requirements for the induction of hepatic microsomal cytochrome P450 by imidazole- and pyridine-containing compounds in rats. J Biochem 1993;114:697-701.

27. Gibson GG, Orton TC, Tamburini PP. Cytochrome P-450 induction by clofibrate Purification and properties of a hepatic cytochrome P-450 relatively specific for the 12- and 11-hydroxylation of dodecanoic acid (lauric acid). Biochem $\mathrm{J}$ 1982;203:161-8.

\title{
XXXVIII Annual Conference of the Indian Pharmacological Society
}

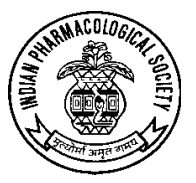

\author{
December 28 - 302005 \\ Madras Medical College, Chennai \\ (Preconference Workshop on 27-12-2005)
}

\section{For further information please contact:}

\author{
Dr. C. B. Tharani
}

Institute of Pharmacology,

Madras Medical College, Chennai.

Ph : 044-25392877- (Phone cum Fax) Mobile: 9884190556

E-mail: ips_38con@yahoo.co.in 